\title{
Seroprevalence and Risk Factors of Toxoplasma gondii Infection among Pregnant Women in Kumasi: A Cross-Sectional Study at a District-Level Hospital, Ghana
}

\author{
Bhavana Singh $\mathbb{D}^{1,2}$ Linda Batsa Debrah, ${ }^{1}$ Godfred Acheampong, ${ }^{3}$ \\ and Alexander Yaw Debrah ${ }^{4}$ \\ ${ }^{1}$ Department of Clinical Microbiology, School of Medicine and Dentistry, Kwame Nkrumah University of Science and Technology, \\ Kumasi, Ghana \\ ${ }^{2}$ University Hospital, Kwame Nkrumah University of Science and Technology, Kumasi, Ghana \\ ${ }^{3}$ Kumasi Centre for Collaborative Research in Tropical Medicine, Kwame Nkrumah University of Science and Technology, \\ Kumasi, Ghana \\ ${ }^{4}$ Faculty of Allied Health Sciences, Kwame Nkrumah University of Science and Technology, Kumasi, Ghana
}

Correspondence should be addressed to Bhavana Singh; littlebhavanasingh@gmail.com

Received 21 November 2020; Revised 4 March 2021; Accepted 22 March 2021; Published 5 April 2021

Academic Editor: David Baker

Copyright () 2021 Bhavana Singh et al. This is an open access article distributed under the Creative Commons Attribution License, which permits unrestricted use, distribution, and reproduction in any medium, provided the original work is properly cited.

\begin{abstract}
Background. This study investigated the prevalence and risk factors of Toxoplasma gondii infection among pregnant women in a district-level hospital in Ghana and compared the diagnostic performance of the rapid diagnostic test (RDT) and enzyme-linked immunosorbent assay (ELISA) for T. gondii diagnosis. Method. This cross-sectional study included 400 consecutive consenting women in their first-trimester stage of pregnancy. A validated well-structured closed-ended questionnaire was used to collect sociodemographic data and possible risk factors of each participant. Blood samples were collected for analysis of $T$. gondii IgG and IgM using the commercial ELISA Kit and RDT. Results. Seroprevalence of toxoplasmosis was $21.5 \%$ and $57.3 \%$ based on the RDT and ELISA technique, respectively. Secondary education ( $\mathrm{cOR}=1.9,95 \% \mathrm{CI}(1.1-3.1)$, and $p=0.020)$ and contact with cats $(\mathrm{cOR}=1.7,95 \% \mathrm{CI}(1.1-2.8)$, and $p=0.030)$ were significant predictors of $T$. gondii infection, with the former being the only independent risk factor for $T$. gondii infection ( $\mathrm{aOR}=1.8,95 \% \mathrm{CI}(1.0-3.0)$, and $p=0.034)$ by the ELISA method. The sensitivity, specificity, and area under the curve (AUC) of RDT-IgM against ELISA were 42.9\%, 95.9\%, and 0.694, respectively, whereas those of RDT-IgG were $31.0 \%, 91.2 \%$, and 0.611 , respectively. The diagnostic consistency between the two methods was fair for both RDT-IgM $(\kappa=0.304)$ and RDT-IgG $(\kappa=0.201)$. Conclusion. The prevalence of T. gondii infection among pregnant women at Kumasi is $21.5 \%$ and $57.3 \%$ based on the RDT and ELISA technique, respectively. Secondary education and contact with cats were the major risk factors of T. gondii infection. Using ELISA as the reference, the RDT used in this study for the diagnosis of T. gondii infection has low sensitivity, and therefore, it is unreliable. However, this finding does not invalidate all RDTs because there are several other brands of RDT with good sensitivity and specificity. Further studies to ascertain the performance of other commercially available RDT kits are needed.
\end{abstract}

\section{Introduction}

Toxoplasmosis is a disease caused by an obligate intracellular protozoan parasite, Toxoplasma gondii (T. gondii). It is widely distributed and can affect humans, pets, and livestock [1]. In a general population, $T$. gondii infection can remain asymptomatic but can also cause lymphadenopathy and flu-like symptoms, which may lead to eye disease, most frequently chorioretinitis [2]. The parasite in its inactive state remains in an individual without presentable signs but flares up upon immunosuppression $[3,4]$.

Pregnant women constitute a specific risk group, and primary infection may be acquired during pregnancy that may lead to abortion, stillbirth, and neurological disorders in the 
TABLE 1: Sociodemographic factors and Toxoplasma gondii seroprevalence.

\begin{tabular}{|c|c|c|c|c|}
\hline \multirow[b]{2}{*}{ Variable } & \multicolumn{2}{|c|}{ T. gondii seroprevalence } & \multirow[b]{2}{*}{$\chi^{2}, \mathrm{df}$} & \multirow[b]{2}{*}{$p$ value } \\
\hline & $\begin{array}{c}\text { Negative (171) } \\
N(\%)\end{array}$ & $\begin{array}{c}\text { Positive (229) } \\
\text { N (\%) }\end{array}$ & & \\
\hline Age (years) & & & $0.97,2$ & 0.616 \\
\hline 20-29 & $73(42.7)$ & $109(47.6)$ & & \\
\hline $30-39$ & $91(53.2)$ & $112(48.9)$ & & \\
\hline 40 and above & $7(4.1)$ & $8(3.5)$ & & \\
\hline Parity & & & $1.02,4$ & 0.907 \\
\hline 0 & $70(40.9)$ & $88(38.4)$ & & \\
\hline 1 & $34(20.5)$ & $56(24.9)$ & & \\
\hline 2 & $41(24.7)$ & $53(23.5)$ & & \\
\hline 3 & $18(10.8)$ & $24(10.6)$ & & \\
\hline$>3$ & $7(4.2)$ & $9(4.0)$ & & \\
\hline Marital status & & & $7.95,2$ & 0.019 \\
\hline Married & $156(91.2)$ & $191(83.4)$ & & \\
\hline Single & $11(6.4)$ & $35(15.3)$ & & \\
\hline Separated & $4(2.3)$ & $3(1.3)$ & & \\
\hline Educational level & & & $9.27,4$ & 0.049 \\
\hline Primary & $9(5.3)$ & $18(7.9)$ & & \\
\hline Junior high & $39(22.8)$ & $59(25.8)$ & & \\
\hline Senior high & $35(20.5)$ & $61(26.6)$ & & \\
\hline Tertiary & $82(48.0)$ & $77(33.6)$ & & \\
\hline No formal education & $6(3.5)$ & $14(6.1)$ & & \\
\hline Income $(N=269)$ & & & $1.13,2$ & 0.588 \\
\hline Low income ( $<\$ 100$ per month) & $66(58.4)$ & $101(64.7)$ & & \\
\hline Middle income ( $\$ 100-400$ per month) & $46(40.7)$ & $54(34.6)$ & & \\
\hline High income ( $>\$ 400$ per month) & $1(0.9)$ & $1(0.6)$ & & \\
\hline Occupation & & & $4.17,4$ & 0.384 \\
\hline Unskilled & $44(25.7)$ & $76(33.2)$ & & \\
\hline Skilled & $33(19.3)$ & $39(17.0)$ & & \\
\hline Self-employed & $35(20.5)$ & $40(17.5)$ & & \\
\hline Professional & $38(22.2)$ & $40(17.5)$ & & \\
\hline Unemployed & $21(12.3)$ & $34(14.8)$ & & \\
\hline Family size $(N=361)$ & & & $2.08,2$ & 0.353 \\
\hline $1-3$ & $89(56.7)$ & $102(50.0)$ & & \\
\hline $4-6$ & $64(40.8)$ & $93(45.6)$ & & \\
\hline$>6$ & $4(2.5)$ & $9(4.4)$ & & \\
\hline
\end{tabular}

unborn child $[5,6]$. Evidence from the literature indicates that the prevalence of $T$. gondii infection among pregnant women ranges from less than $1 \%$ to $92 \%$ depending on the geographical location $[2,4,7]$. Among African populations, studies in Tanzania [8] and Ghana [9] reported T. gondii seroprevalence of $30.9 \%$ and $92.5 \%$, respectively, among pregnant women, and factors such as eating undercooked or cured meat, having a cat as a pet, low educational level, contact with soil, crowded conditions, parity, and consumption of raw vegetables were noted as predisposing factors $[8,9]$.

More so, a higher prevalence is observed in tropical countries with a humid and warm climate, and conversely, a lower prevalence is found in colder countries. Several anthropogenic factors explain a large part of the variations in human sero- prevalence such as dietary habits in terms of the method of cooking meat, kinds of meat or vegetables consumed, and vegetable cleaning; handwashing techniques adopted by a community; economic, social, or cultural habits; quality of water; and sanitation coverage [10-12]. Also, seroprevalence increases with age and the rate of acquiring infection in relation to age varies according to the country and socioeconomic level. Seroprevalence in children living under poor hygienic conditions is high, probably linked to telluric or waterborne contamination by oocyst ingestion. This implies that water is an important source of human infection in areas where humans use unfiltered surface water for consumption [10-12].

The burden of T. gondii infection is reported to be generally severe in immunocompromised populations such as 
TABLE 2: Hygienic predisposing factors and Toxoplasma gondii seroprevalence.

\begin{tabular}{|c|c|c|c|c|}
\hline \multirow{2}{*}{ Variable } & \multicolumn{2}{|c|}{ T. gondii seroprevalence } & \multirow[b]{2}{*}{$\chi^{2}, \mathrm{df}$} & \multirow[b]{2}{*}{$p$ value } \\
\hline & $\begin{array}{c}\text { Negative } \\
N(\%)\end{array}$ & $\begin{array}{c}\text { Positive } \\
N(\%)\end{array}$ & & \\
\hline Source of water & & & $1.22,1$ & 0.285 \\
\hline Pipe-borne & $109(63.7)$ & $158(69.0)$ & & \\
\hline Borehole & $62(36.3)$ & $71(31.0)$ & & \\
\hline Waste disposal facility & & & $3.11,2$ & 0.211 \\
\hline WC & $123(74.5)$ & $145(66.2)$ & & \\
\hline Public toilets & $41(24.8)$ & $72(32.9)$ & & \\
\hline Land, rivers, and streams & $1(0.6)$ & $2(0.9)$ & & \\
\hline Ingestion of raw vegetables & & & $1.19,1$ & 0.332 \\
\hline No & $23(13.5)$ & $40(17.50)$ & & \\
\hline Yes & $148(86.5)$ & $189(82.5)$ & & \\
\hline Ingestion of uncooked meat & & & $0.02,1$ & 0.916 \\
\hline No & $61(35.7)$ & $80(34.9)$ & & \\
\hline Yes & $110(64.3)$ & $149(65.1)$ & & \\
\hline Cleaning cooking materials & & & $1.40,1$ & 0.258 \\
\hline No & $6(3.5)$ & $14(6.1)$ & & \\
\hline Yes & $165(96.5)$ & $215(93.9)$ & & \\
\hline Meat handling (e.g., beef mutton) & & & $0.38,1$ & 0.558 \\
\hline No & $21(12.3)$ & $33(14.4)$ & & \\
\hline Yes & $150(87.7)$ & $196(85.6)$ & & \\
\hline Contact with soil & & & $1.35,1$ & 0.262 \\
\hline No & $104(60.8)$ & $126(55.0)$ & & \\
\hline Yes & $67(39.2)$ & $103(45.0)$ & & \\
\hline Owning pets & & & $1.13,1$ & 0.339 \\
\hline No & $117(68.4)$ & $145(63.3)$ & & \\
\hline Yes & $54(31.6)$ & $84(36.7)$ & & \\
\hline Owning cats & & & $3.39,1$ & 0.083 \\
\hline No & $148(86.5)$ & $182(79.5)$ & & \\
\hline Yes & $23(13.5)$ & $47(20.5)$ & & \\
\hline Contact with cats & & & $4.79,1$ & 0.027 \\
\hline No & $140(81.9)$ & $166(72.5)$ & & \\
\hline Yes & $31(18.1)$ & $63(27.5)$ & & \\
\hline Housing of cats & & & $1.11,1$ & 0.304 \\
\hline No & $143(83.6)$ & $182(79.5)$ & & \\
\hline Yes & $28(16.4)$ & $47(20.5)$ & & \\
\hline Litter box for cats & & & $2.99,1$ & 0.109 \\
\hline No & $153(89.5)$ & $191(83.4)$ & & \\
\hline Yes & $18(10.5)$ & $38(16.6)$ & & \\
\hline
\end{tabular}

pregnant women where studies have linked this condition to severe pregnancy outcomes $[5,13]$. Infection with $T$. gondii in early pregnancy often leads to severe consequences such as abortion and abnormalities, as compared to the asymptomatic presentation of the newborn when infected during the third trimester [14].

Due to the detrimental effects of $T$. gondii infection on the mother and her unborn baby, early diagnosis of the infection is key. There are several laboratory tests for the diagnosis of $T$. gondii, including the rapid diagnostic test (RDT), sero- logical tests (such as enzyme-linked immunosorbent assay (ELISA)), PCR, and histological and cytological examination of tissue and body fluids [15]. RDT is easy to perform, is rapid, and does not need trained personnel and special equipment, and therefore, it is suitable for convenient testing [16]. For this reason, most diagnostic services in some African countries such as Ghana rely on RDT. However, most RDT kits on the market have not been field-evaluated. Moreover, the unreliability of RDT for T. gondii diagnosis has been reported [14]. Thus, other relatively less expensive tests like 


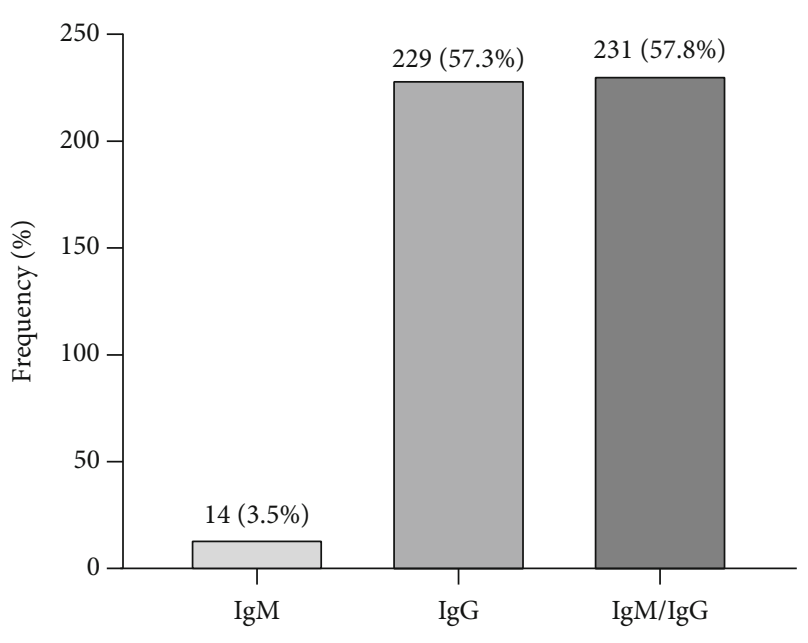

FIGURE 1: Seroprevalence of Toxoplasma gondii antibodies among the study participants by ELISA. Percentages were calculated from the number of positives over the total number of participants.

ELISA are being advocated. One advantage of ELISA is its ability to detect specific anti-Toxoplasma IgG and IgM antibodies with higher accuracy compared to RDT, although skilled personnel is required [16].

This study is thus aimed at assessing the prevalence and risk factors of $T$. gondii infection among pregnant women attending antenatal care at the Kwame Nkrumah University of Science and Technology (KNUST) Hospital in Ghana and comparing the diagnostic performance of the RDT and ELISA technique for $T$. gondii diagnosis.

\section{Materials and Methods}

2.1. Study Design/Setting. This cross-sectional study, which was part of a larger longitudinal study, was conducted in Kumasi Metropolis at the Kwame Nkrumah University of Science and Technology (KNUST) Hospital from August 2017 to February 2018. The KNUST Hospital caters to a population of over 200,000 per annum, and it is made of 21,000 students, 30,000 staff and dependents, and about 150,000 people from over 30 surrounding communities. The University Health Services offers services in general medical care as well as specialist services.

2.2. Sample Size. The sample size for the study was calculated using Fischer's sampling formula $\left(N=Z^{2} P Q / d^{2}\right)$, where $Z$ is the critical value of the normal distribution (1.96 at $95 \% \mathrm{CI}) ; P$ is the estimated prevalence of $T$. gondii among pregnant women in Ghana (51.2\%) [17]; $Q=$ $100-P$; and $d$ is the absolute precision or sampling error tolerated $=5 \%$. From the above equation, the minimum sample size for this study was 384 . However, in an effort to increase the statistical power of the study, we recruited 400 consecutive consenting pregnant women attending antenatal care at the KNUST Hospital.

2.3. Ethical Approval. Ethical approval was obtained from the Committee on Human Research Publication and Ethics
(CHRPE) of the School of Medicine and Dentistry of the Kwame Nkrumah University of Science and Technology, Kumasi (CHRPE/AP/541/17). Informed consent, either signed or thumbed, was obtained from every participant before enrolling them in this study.

2.4. Questionnaire Administration. A validated questionnaire, designed by reviewing previous studies of similar objectives, was used to collect information on sociodemographic factors such as age, marital status, level of education, income, behavioral practices including eating and handling of meat, contact with cats and level of handling cats or cat litter, and obstetric history in terms of parity which is the number of times a woman has given birth.

2.5. Sampling Procedure. Two millimeters $(2 \mathrm{ml})$ of venous blood was drawn from each participant. The blood samples were transported to the Kumasi Centre for Collaborative Research (KCCR) laboratory in an ice chest. Plasma was prepared from the whole blood sample by spinning it at $1500 \mathrm{rpm}$ for $10 \mathrm{~min}$, and aliquots were stored at $-20^{\circ} \mathrm{C}$ until subsequent analysis.

2.6. Sample Analysis. Each sample was tested for the presence of Toxoplasma antibodies using both the rapid diagnostic test (RDT) IgG and IgM kits (Innovation Biotech TOXO IgG/IgM RDT, Beijing, China) and the commercial ELISA Kit (INVBIO IgG and IgM ELISA, Innovation Biotech Beijing Co. Ltd., China) following the manufacturer's instructions. T. gondii antigen detection was performed by the sandwich ELISA method (INVBIO IgG and IgM ELISA, Innovation Biotech Beijing Co. Ltd., China) according to the manufacturer's instructions. Briefly, all reagents were allowed to attain room temperature before use. The wash buffers were diluted at a 1:40 ratio of distilled water before use. One hundred microliters $(100 \mu \mathrm{l})$ of the sample dilution liquid was added in each well, and $10 \mu \mathrm{l}$ of the sample was added. The resultant solution was mixed thoroughly, covered with an adhesive cover, and incubated at $37^{\circ} \mathrm{C}$ for $20 \mathrm{~min}$. After incubation, the mixture was aspirated from the wells followed by four washes with the wash solution. Residual wash solution droplets were removed by blotting the microtitre plate onto an absorbent paper. A $50 \mu \mathrm{l}$ conjugate was added and incubated at $37^{\circ} \mathrm{C}$ for 20 minutes, and a $100 \mu \mathrm{l}$ of tetramethylbenzidine (TMB) solution (prepared from $50 \mu \mathrm{l}$ of chromogen $\mathrm{A}$ and $50 \mu \mathrm{l}$ of chromogen $\mathrm{B}$ ) was pipetted into each well, mixed gently, and incubated at $37^{\circ} \mathrm{C}$ for $10 \mathrm{~min}$. Fifty microliters $(50 \mu \mathrm{l})$ of Stop Solution was added to each well and gently mixed for $30 \mathrm{~s}$ to stop the reaction. The absorbance of each well was measured spectrophotometrically at $450 \mathrm{~nm}$ using a Thermo Electron Multiskan EX plate reader (Shanghai, China).

2.7. Data Analysis. Statistical analysis was done using a statistical package SPSS (version 25). To compare the diagnostic consistency between RDT and ELISA, Cohen's kappa coefficient $(\kappa)$ was used. Diagnostic accuracy tests including sensitivity, specificity, positive predictive value (PPV), negative predictive value (NPV), and area under the curve (AUC) were performed using ELISA as the reference. 


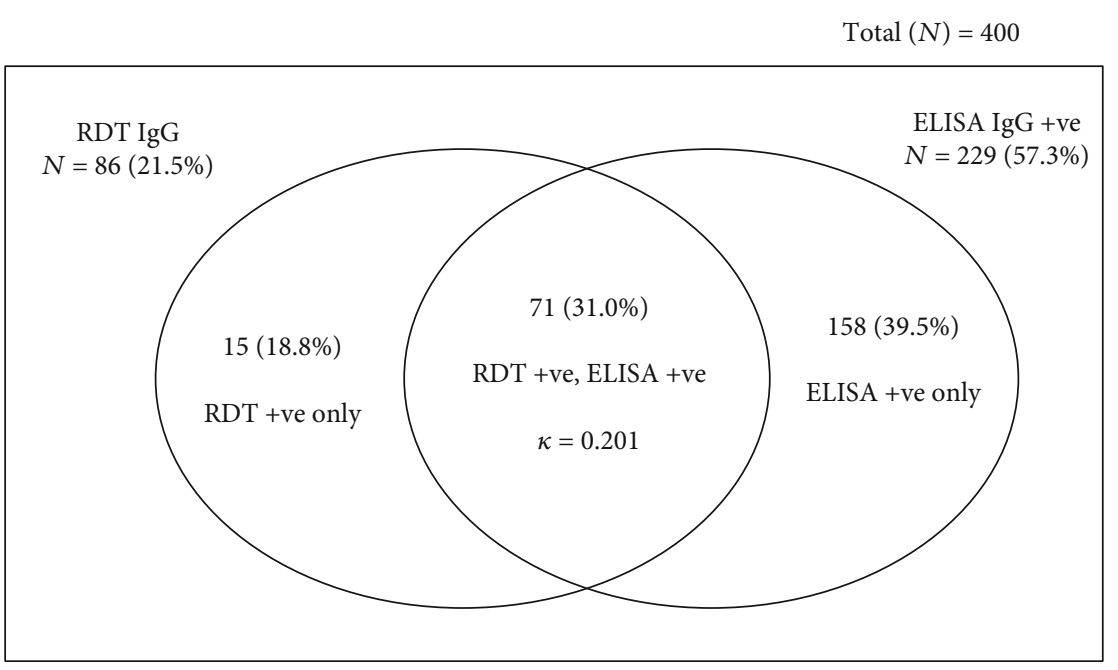

FIGURE 2: Seroprevalence of Toxoplasma gondii antibodies (IgG) among the study participants. The number of IgG-positive cases based on RDT was compared with ELISA using kappa statistics.

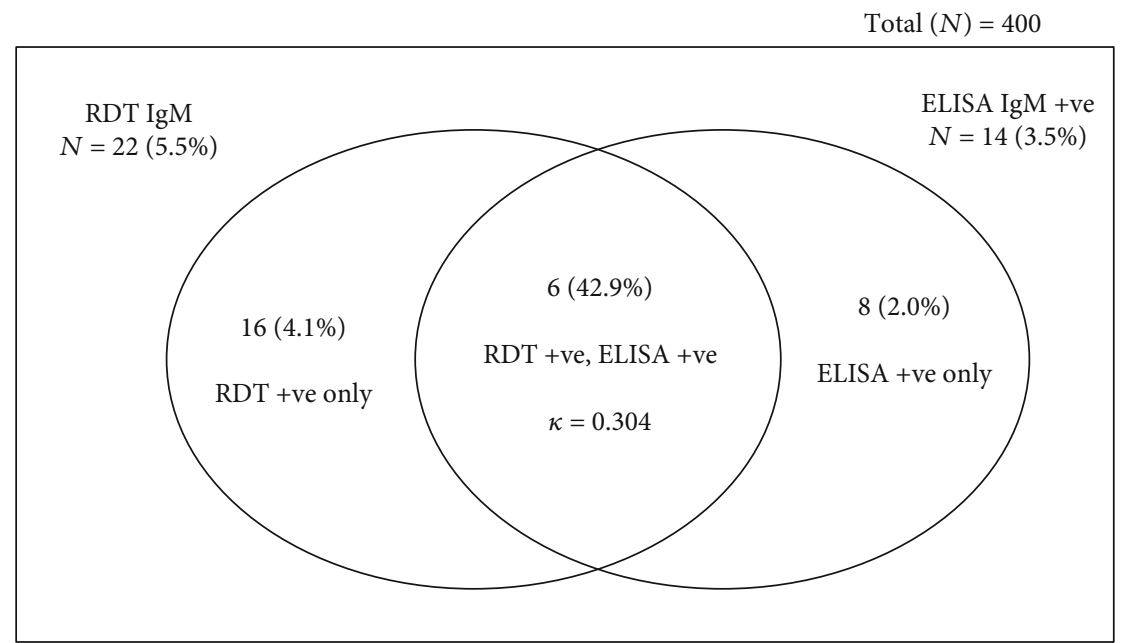

FIGURE 3: Seroprevalence of Toxoplasma gondii antibodies (IgM) among the study participants. The number of IgM-positive cases based on RDT was compared with ELISA using kappa statistics.

The associations between T. gondii infection and possible risk factors were tested with a chi-squared test. The magnitude of associations was assessed using odds ratios (OR) at a 95\% confidence interval (CI). A multivariate logistic regression model was used to identify the explanatory variables among confounding risk variables that would explain the occurrence of toxoplasmosis. A $p$ value $<0.05$ was considered statistically significant.

\section{Results}

3.1. Demographic Characteristics of the Study Participants. Approximately $51.0 \%$ of the study participants were 30 39 years old. A higher proportion of the participants (37.5\%) had no prior children; $41.8 \%$ had low-income salaries $(<\$ 100$ per month); $47.8 \%$ had a family size of $1-3$; $30 \%$ were unskilled workers; and $39.8 \%$ had completed tertiary education. The majority $(86.6 \%)$ of the study subjects were married (Table 1).
A higher percentage of the study participants used pipeborne water $(67.0 \%)$ and water closest for their organic waste disposal (67.3\%). In terms of cooking and eating practices, a major percentage $(84.3 \%)$ of the participants ingested raw vegetables, about $36.2 \%$ of the study participants eat raw meat without cooking, $95.0 \%$ used clean cooking surfaces and utensils after contact with risk materials, $86.5 \%$ handled meat, and $42.5 \%$ have constant contact with soil. In terms of pet handling practices among seropositive participants, $36.7 \%$ owned pets and $20.5 \%$ owned cats. About $27.5 \%$ had contact with cats, and $83.4 \%$ did not have a litter box for cats. Among subjects that had a litter box for cats, a major proportion $(85.7 \%)$ did not use gloves in handling it and $67.9 \%$ did not practice handwashing after contact with cats (Table 2).

3.2. Toxoplasma gondii Seroprevalence and Associated Factors. Overall, the seroprevalence of IgM, IgG, and IgM/IgG $T$. gondii antibodies by ELISA was 3.5\%, 57.3\%, and $57.8 \%$, respectively (Figure 1 ). 
TABLE 3: Bivariate and multivariate analyses of risk factors significantly associated with Toxoplasma gondii seroprevalence in this present study.

\begin{tabular}{|c|c|c|c|c|c|c|}
\hline \multirow{2}{*}{ Risk factors } & \multirow{2}{*}{ Number tested } & \multirow{2}{*}{$\%$ positives } & \multicolumn{2}{|c|}{ Univariate } & \multicolumn{2}{|c|}{ Multivariate } \\
\hline & & & OR $(95 \% \mathrm{CI})$ & $p$ value & OR $(95 \% \mathrm{CI})$ & $p$ value \\
\hline \multicolumn{7}{|l|}{ Marital status } \\
\hline Married & 347 & 55.0 & $1.6(0.4-7.4)$ & 0.525 & $1.4(0.3-6.6)$ & 0.640 \\
\hline Single & 46 & 76.1 & $4.2(0.8-21.9)$ & 0.085 & $3.4(0.6-18.1)$ & 0.149 \\
\hline Separated & 7 & 42.9 & 1 (reference) & & 1 (reference) & \\
\hline \multicolumn{7}{|l|}{ Educational level } \\
\hline No formal education & 20 & 70.0 & $2.5(0.9-6.8)$ & 0.076 & $2.6(0.9-7.1)$ & 0.066 \\
\hline Primary & 27 & 66.7 & $2.1(0.9-5.0)$ & 0.084 & $2.0(0.8-4.7)$ & 0.121 \\
\hline Junior high & 98 & 60.2 & $1.6(1.0-2.7)$ & 0.067 & $1.5(0.9-2.6)$ & 0.107 \\
\hline Senior high & 96 & 63.5 & $1.9(1.1-3.1)$ & 0.020 & $1.8(1.0-3.0)$ & 0.034 \\
\hline Tertiary & 156 & 48.4 & 1 (reference) & & 1 (reference) & \\
\hline \multicolumn{7}{|l|}{ Owning cats } \\
\hline No & 330 & 55.2 & 1 (reference) & & 1 (reference) & \\
\hline Yes & 70 & 67.1 & $1.7(1.0-2.9)$ & 0.067 & $1.3(0.6-2.6)$ & 0.517 \\
\hline \multicolumn{7}{|l|}{ Contact with cats } \\
\hline No & 306 & 54.2 & 1 (reference) & & 1 (reference) & \\
\hline Yes & 94 & 67.0 & $1.7(1.1-2.8)$ & 0.030 & $1.3(0.7-2.4)$ & 0.475 \\
\hline
\end{tabular}

Out of the 400 pregnant women screened for T. gondii IgG, $86(21.5 \%)$ were positive with RDT and $229(57.3 \%)$ were positive for ELISA. Upon stratification by the antibody type, the percentage of the participants detected by RDT-IgG only as positives was $8.8 \%$ and that by ELISA only was $39.5 \%$ (Figure 2). Also, the percentage of IgM positives detected by RDT alone was $4.1 \%$ while that by ELISA was $2.0 \%$ (Figure 3).

Secondary education $(\mathrm{cOR}=1.9,95 \% \mathrm{CI}(1.1-3.1)$, and $p=0.020)$ and contact with cats $(\mathrm{cOR}=1.7,95 \% \mathrm{CI}(1.1-$ 2.8 ), and $p=0.030$ ) were significant predictors of $T$. gondii infection, with the former being the only independent risk factor for $T$. gondii infection $(\mathrm{aOR}=1.8,95 \%$ CI (1.0-3.0), and $p=0.034$ ) by the ELISA method (Table 3 ).

3.3. Validation of the Rapid Diagnostic Test Using the ELISA Technique. The sensitivity and specificity of RDT-IgG using ELISA as the gold standard were $36 \%$ and $91.2 \%$. There was a fair diagnostic agreement (kappa $=0.201)$ between the RDT-IgG and ELISA IgG tests. The sensitivity and specificity of RDT-IgM were $43 \%$ and $96 \%$ with a fair diagnostic agreement $(\mathrm{kappa}=0.304)$. The effectiveness of RDT-IgM $(\mathrm{AUC}=0.694)$ compared with RDT-IgG $(\mathrm{AUC}=0.611)$ was better using ELISA as the gold standard (Figure 4 and Table 4).

\section{Discussion}

This study investigated the prevalence of T. gondii infection among pregnant women in Kumasi Metropolis using the commercially available rapid diagnostic test (RDT) and ELISA test kits that detect anti-Toxoplasma IgG and IgM antibodies. Infection with T. gondii in early pregnancy presents the risk of fetal transmission, where the rates of trans- mission range between $60 \%$ and $81 \%$ in the third trimester $[15,16]$. Classically, congenital infection results from primary acquired maternal infection during gestation. The severity of fetal infection is inversely correlated to the stage at which infection occurs, and $80 \%$ of neonates are asymptomatic when infected during the third trimester of gestation [14]. However, in the first-trimester transplacental transmission, the consequences for fetal development are heavy and severe, often leading to severe abnormalities or to abortion. Neonatal manifestations of congenital toxoplasmosis have been related to fetal conditions, including hydrocephalus, microcephaly, intracranial calcifications, retinochoroiditis, strabismus, blindness, epilepsy, and psychomotor and mental retardation [17-19]. Hence, a timely method of detection of $T$. gondii infection in pregnant women is key.

The result showed that more than half of the study participants had an overall IgG- and IgM-positive infection (57.8\%), indicative of acute and previous exposure. T. gondii IgG seropositivity only was $57.3 \%$ in our study, indicative of past or previous exposure. Even though the seroprevalence was high in this study, it was relatively low as compared to the $95.9 \%$ detected by Ayi et al. [9] in Accra, in southern Ghana. In our study, T. gondii IgM positivity was found in only $3.5 \%$ of our participants and all other seropositive cases were IgG, indicating chronic infection. Several other similar studies have reported no or few IgM positivity compared to IgG [18]. In line with our T. gondii IgM seropositivity, a systematic review by Bigna et al. [36] indicated a global IgM seroprevalence of $1.9 \%$, with the highest being in Eastern Mediterranean (4.1\%) and America being the lowest in $T$. gondii IgM (1.1\%) seroprevalence. Moreover, global IgG seroprevalence was found to be $32.9 \%$ which is lower than our findings. In Srilanka, however, Iddawela et al. [37] found a T. gondii-specific IgM seropositivity as low as $0.3 \%$ and IgG 

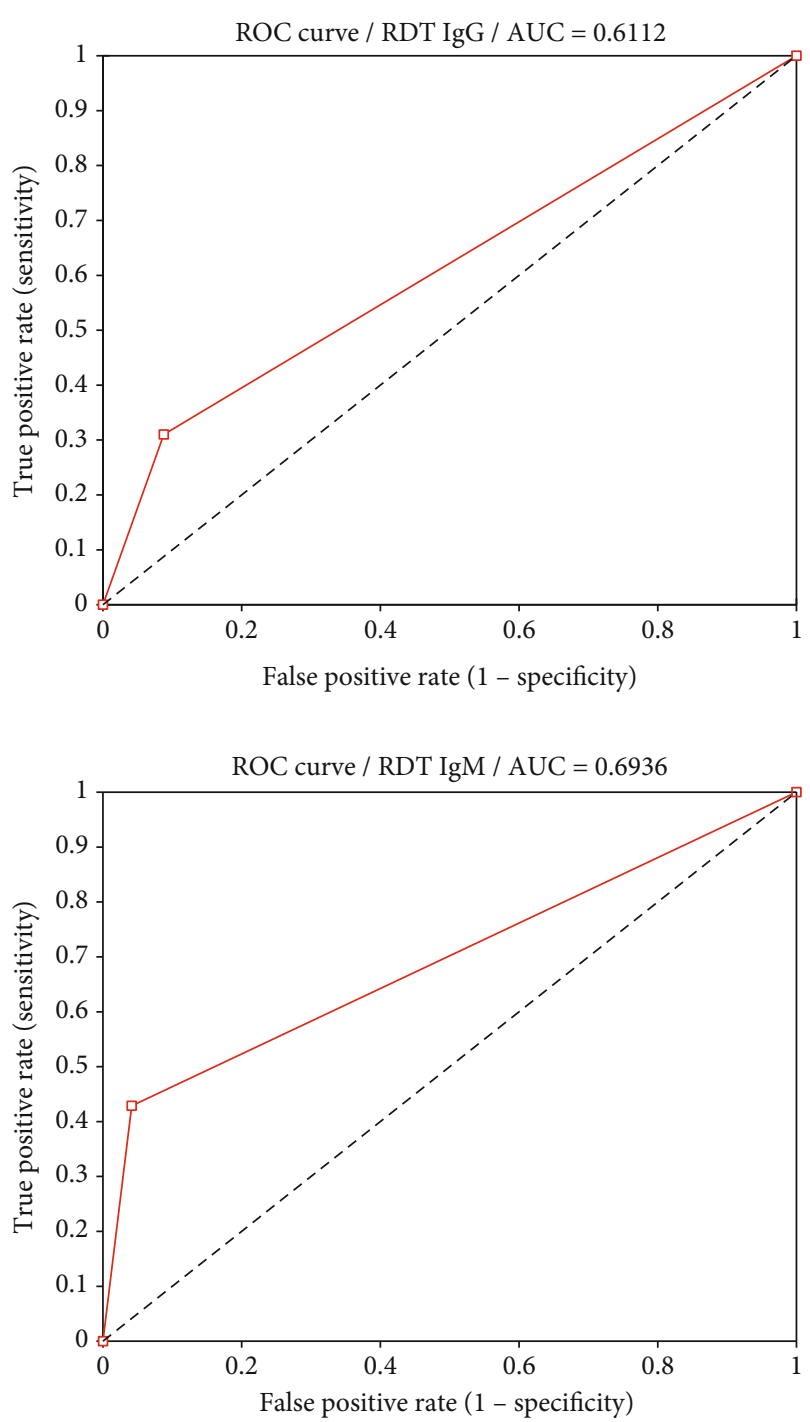

FIGURE 4: Receiver operating characteristic (ROC) curve showing the diagnostic consistency.

seropositivity far lower than our finding, that is, $29.9 \%$, indicating that almost $70.1 \%$ of their population are susceptible to primary acute infection during pregnancy and possible fetal anomalies. In patients with acute infection, T. gondiispecific IgM appears initially and antibody levels become negative in few months. However, persistent IgM positivity in chronic stages of Toxoplasma infection has been reported [19]. Thus, the presence of $\operatorname{IgM}$ antibodies does not always confirm acute infection. However, the negative Toxoplasma IgM test rules out recently acquired Toxoplasma infection [20]. In antibody detection for diagnosis of toxoplasmosis, anti-IgM suggests recent, acute, or ongoing infections and IgM antibodies indicate acute infections because these antibodies are not usually in acquired immunity and are very rare in chronic infections $[9,21]$.

This study is the first of its kind in Kumasi Metropolis, and the high seroprevalence is not surprising as human infection is widely reported, with nearly one-third of the world population being exposed to the parasite [22]. As the location is an urban area and not a rural one, toxoplasmosis is not exclusive to marginalized communities. In high-income countries, like the USA and the UK, it has been estimated that between $10 \%$ and $40 \%$ of the population is infected, while in Central and South America and continental Europe, the prevalence ranges from $50 \%$ to $80 \%$ [23]. In our study, IgM antibodies were detected in $3.5 \%$ of the study population, implying the possible presence of acute infection.

Importantly, in this study, there was an association between contact with cat and seropositivity of Toxoplasma, consistent with reports by previous studies $[9,13]$. Cats have been implicated in the transmission of $T$. gondii infection. Owning cats and subsequent exposure to their feces facilitate the transmission of the parasite [9]. Congruently, Al Hamdani and Mahdi [13] also found that Toxoplasma antibodies were more prevalent in pregnant women with cats at home than in pregnant women that did not own cats. Iddawela et al. [37] in Srilanka, on the other hand, did not find the association between $T$. gondii seropositivity and owning a cat, but rather preparation and selling raw meat $(p=0.05)$ and household gardening $(p=0.01)$ were significantly associated with being seropositive with $T$. gondii.

We also found low educational status to be associated with higher T. gondii seroprevalence, which is consistent with a study by Kwakye-Nuako in Ghana [24] who also found a higher prevalence of $T$. gondii infection among secondary school graduates and participants with no education as compared to university graduates. Furthermore, being married was also associated with a higher seroprevalence $T$. gondii infection and this is in line with a study by Singh et al. [25]. Frimpong et al. [39] also found that being a farmer and being involved in construction work showed a 15.5 times likelihood of contracting the infection in Lusaka, as well as the socioeconomic status of the pregnant women that had an inverse relationship with infection. There is an extent of diversity when it comes to the level of education and occupation, as different studies investigate different economic activities under this term and others do not specify exactly which activities were investigated.

Eating uncooked meat and handling of meat were not significantly associated with toxoplasmosis in our study, and it is in line with Frimpong et al. [39] in Lusaka. However, several other studies have reported handling of raw meat and ingestion of undercooked meat to be associated with toxoplasmosis $[37,40]$. The definition of uncooked meat may differ among these studies due to different settings, such as culture and preference, under which these studies were carried out. This study considered uncooked meat to be meat not adequately cooked for more than 30 minutes. This is, however, not a documented universal standard since undercooked meat in some European countries refers to raw meat which still has blood in it, and this is uncommon in our setting. However, the criteria other studies used to define undercooked meat in their studies are unclear, which could explain why this variable did not come out as an important determining factor of the infection in this study.

In comparing the diagnostic utility of RDT and ELISA, RDT-IgM performed better in diagnosing T. gondii infection than RDT-IgG. This finding is in harmony with a study by 
TABLE 4: Diagnostic performance of T. gondii RDT-IgG and RDT-IgM diagnostic tests against the ELISA technique.

\begin{tabular}{|c|c|c|c|c|c|c|c|}
\hline Diagnostic test & Sensitivity & Specificity & Accuracy & PPV & NPV & Cohen's kappa & AUC \\
\hline RDT-IgG & 0.31 & 0.912 & 0.56 & 0.38 & 0.83 & 0.201 & 0.611 \\
\hline RDT-IgM & 0.43 & 0.960 & 0.94 & 0.27 & 0.97 & 0.304 & 0.694 \\
\hline
\end{tabular}

NPV: negative predictive value; PPV: positive predictive value; AUC: area under the curve.

Bassiony et al. [26]. Contrary to our results, Huang et al. [27] reported a high RDT sensitivity and specificity of 97.2 and $95.8 \%$, respectively, in the detection of anti-T. gondii antibodies in the cat's sera. The relative agreement was $96.1 \%$ between RDT and ELISA. The disparity between our findings and that of Huang et al. could be due to the differences in the type of material tested (human sample versus cat sample). It is important to note that despite the advantage of the RDT test of being rapid and easy to perform and requiring no special expertise or high technological machines to adjudge positive or negative results, a higher concentration and purity of the antigen are required for this test [28], and sensitivity is most often challenged. Notwithstanding the low sensitivity recorded by the RDT used in this study, there are several other commercial RDTs that have demonstrated higher sensitivities, and therefore, their importance and efficiency for mass screening cannot be completely overlooked considering the convenience and cost benefits of RDT in resource-poor countries [37].

Overall, this study has shown a moderate to high seroprevalence of $T$. gondii infection among pregnant women in Kumasi. However, these results cannot be generalized to the whole country as it focused on females attending the ANC at the KNUST Hospital, mostly a semiurban educated population. Levels of exposure to toxoplasmosis could however vary in more rural areas where involvement in agricultural activities and soil contact is greater. Therefore, more studies need to be done in other areas in a larger population with sociodemographic variation to represent the whole country. Also of note, only one brand of RDT was utilized in this study and the sensitivity/specificity may vary when a different brand is used. We recommend that future studies take other brands of RDT used in the country into consideration. Furthermore, the few studies conducted in Ghana showed moderate to high seroprevalence in the population $[9,29]$. Moreover, since congenital toxoplasmosis can only occur in pregnant women primarily infected with $T$. gondii during pregnancy, the serological status at the beginning of pregnancy is very important. Women who are seropositive in their first trimester have a limited risk of giving birth to babies with congenital toxoplasmosis, and hence, preventive measures can help reduce the risk of infection during pregnancy in seronegative women. There is therefore the need to follow up the seroconversion of seronegative mothers for the next two trimesters to identify women with recent Toxoplasma infection and to detect the possible transmission rate to the babies born from the seroconverted mothers.

\section{Conclusion}

The prevalence of $T$. gondii infection among pregnant women at Kumasi is $21.5 \%$ and $57.3 \%$ based on the RDT and ELISA technique, respectively. Secondary education and contact with cats were the major risk factors of $T$. gondii infection. Using ELISA as the gold standard, our brand of RDT for the diagnosis of T. gondii infection is less reliable. However, this finding does not invalidate all Toxoplasma RDTs because there are several other brands of RDT with good sensitivity and specificity. Further studies to ascertain the performance of other commercially available RDT kits are needed.

We recommend Toxoplasma screening at antenatal clinics in Ghana. Since T. gondii infection is acquired predominantly from the environment, health education of the pregnant women will be an inexpensive but significant mode of reducing the risk of acquisition and transmission of the infection.

\section{Data Availability}

The datasets used and/or analyzed during the current study are available from the corresponding author on reasonable request.

\section{Conflicts of Interest}

The authors declare that they have no competing interests.

\section{Authors' Contributions}

BS, LBD, and AYD conceived and designed the study. LBD and AYD supervised the research and laboratory analysis. BS and GA were involved in the collection of data and laboratory analysis. BS, LBS, and AYD were involved in the statistical analysis and interpretation, drafting, and revision of the manuscript. All authors read and approved the final manuscript.

\section{Acknowledgments}

We are grateful to the Department of Clinical Microbiology, KNUST, Kumasi, Ghana, Kumasi Centre of Collaborative Research, KNUST, Kumasi, Ghana, the Director, University Health Services, and the staff of the KNUST Hospital, Mr. Evans Adu Asamoah and Eddie-Williams Owiredu from the Department of Molecular Medicine, KNUST, Kumasi, Ghana, and my colleague Mr. Kwabena Dankwa from the Department of Clinical Microbiology, School of Medicine and Dentistry, KNUST, Kumasi, Ghana, for their help with the collection and processing of data. This study was partly funded by KNUST-BSU III. 


\section{References}

[1] G. Saadatnia and M. Golkar, "A review on human toxoplasmosis," Scandinavian journal of infectious diseases., vol. 44, no. 11, pp. 805-814, 2012.

[2] A. P. LOPES, J. P. DUBEY, O. MOUTINHO et al., "Seroepidemiology of Toxoplasma gondii infection in women from the north of Portugal in their childbearing years," Epidemiology \& Infection, vol. 140, no. 5, pp. 872-877, 2012.

[3] A. M. Tenter, A. R. Heckeroth, and L. M. Weiss, “_Toxoplasma gondii_: from animals to humans," International Journal for Parasitology, vol. 30, no. 12-13, pp. 1217-1258, 2000.

[4] G. Pappas, N. Roussos, and M. E. Falagas, “Toxoplasmosis snapshots: global status of _Toxoplasma gondii_seroprevalence and implications for pregnancy and congenital toxoplasmosis," International Journal for Parasitology, vol. 39, no. 12, pp. 1385-1394, 2009.

[5] P. R. Torgerson and P. Mastroiacovo, "The global burden of congenital toxoplasmosis: a systematic review," Bulletin of the World Health Organization., vol. 91, no. 7, pp. 501-508, 2013.

[6] N. Tekkesin, "Diagnosis of toxoplasmosis in pregnancy: a review," HOAJ Biology., vol. 1, no. 1, p. 9, 2012.

[7] R. S. Vaz, V. Thomaz-Soccol, E. Sumikawa, and A. T. B. Guimarães, "Serological prevalence of Toxoplasma gondii antibodies in pregnant women from Southern Brazil," Parasitology research., vol. 106, no. 3, pp. 661-665, 2010.

[8] B. Mwambe, S. E. Mshana, B. R. Kidenya et al., "Sero-prevalence and factors associated with Toxoplasma gondii infection among pregnant women attending antenatal care in Mwanza, Tanzania," Parasites \& vectors, vol. 6, no. 1, p. 222, 2013.

[9] I. Ayi, S. Edu, K. A. Apea-kubi, D. Boamah, K. M. Bosompem, and D. Edoh, "Sero-epidemiology of toxoplasmosis amongst pregnant women in the Greater Accra Region of Ghana," Ghana medical journal, vol. 43, no. 3, 2010.

[10] J. L. Jones and J. P. Dubey, "Waterborne toxoplasmosis recent developments," Experimental parasitology, vol. 124, no. 1, pp. 10-25, 2010.

[11] A. Retmanasari, B. S. Widartono, M. A. Wijayanti, and W. T. Artama, "Prevalence and risk factors for toxoplasmosis in Middle Java, Indonesia," EcoHealth, vol. 14, no. 1, pp. 162170, 2017.

[12] G. S. A. Smit, B. T. L. Vu, D. T. Do et al., "Sero-epidemiological status and risk factors of toxoplasmosis in pregnant women in Northern Vietnam," BMC infectious diseases, vol. 19, no. 1, p. 329, 2019.

[13] M. M. Al Hamdani and N. K. Mahdi, "Toxoplasmosis among women with habitual abortion," East Mediterrean health journal, vol. 3, pp. 310-315, 1997.

[14] F. Hassaneina and A. I. Shehata, "Rapid immunochromatographic test (RDT) versus ELISA technique for diagnosing toxoplasmosis among individuals with mental disabilities," International Journal of Innovative Research \& Development, vol. 7, pp. 61-66, 2018.

[15] P. A. Moncada and J. G. Montoya, "Toxoplasmosis in the fetus and newborn: an update on prevalence, diagnosis and treatment," Expert Review of Anti-infective Therapy, vol. 10, no. 7, pp. 815-828, 2014.

[16] F. Robert-Gangneux and M.-L. Darde, "Epidemiology of and diagnostic strategies for toxoplasmosis," Clinical Microbiology Reviews, vol. 25, no. 2, pp. 264-296, 2012.
[17] I. Ayi, A. O.-K. Sowah, E. A. Blay, T. Suzuki, N. Ohta, and P. F. Ayeh-Kumi, "Toxoplasma gondii infections among pregnant women, children and HIV-seropositive persons in Accra, Ghana," Tropical medicine and health, vol. 44, no. 1, 2016.

[18] V. Nissapatorn, T. H. Leong, R. Lee, I. J. Init-Ithoi, and T. S. Yen, "Seroepidemiology of toxoplasmosis in renal patients," Southeast Asian journal of tropical medicine and public health, vol. 42, no. 2, pp. 237-247, 2011.

[19] O. Liesenfeld, C. Press, J. G. Montoya et al., "False-positive results in immunoglobulin $\mathrm{M}(\mathrm{IgM})$ toxoplasma antibody tests and importance of confirmatory testing: the Platelia Toxo IgM test," Journal of clinical microbiology., vol. 35, no. 1, pp. 174178, 1997.

[20] S. D. L. P. Subasinghe, N. D. Karunaweera, A. Kaluarachchi et al., "Toxoplasma gondii seroprevalence among two selected groups of women," Sri Lankan Journal of Infectious Diseases, vol. 1, no. 1, p. 9, 2011.

[21] M. H. Bessieres, C. Roques, A. Berrebi, V. Barre, M. Cazaux, and J. P. Seguela, "IgA antibody response during acquired and congenital toxoplasmosis," Journal of Clinical Pathology, vol. 45, no. 7, pp. 605-608, 1992.

[22] J. M. Furtado, J. R. Smith, R. Belfort, D. Gattey, and K. L. Winthrop, "Toxoplasmosis: a global threat," Journal of global infectious diseases, vol. 3, no. 3, pp. 281-284, 2011.

[23] Y. Sukthana, "Toxoplasmosis: beyond animals to humans," Trends in Parasitology, vol. 22, no. 3, pp. 137-142, 2006.

[24] G. Kwakye-Nuako, P. Osei-Owusu, D. Amoako-Sakyi, D. Obiri-Yeboah, P. Ayeh-Kumi, and C. Muñoz-Zanzi, “Toxoplasmosis in Ghana: seroprevelance study in the coastal population," International society for infectious diseases, pp. 9-10, 2010.

[25] S. Singh, A. Munawwar, S. Rao, S. Mehta, and N. K. Hazarika, "Serologic prevalence of Toxoplasma gondii in Indian women of child bearing age and effects of social and environmental factors," PLoS Neglected Tropical Diseases, vol. 8, no. 3, article e2737, 2014.

[26] H. Bassiony, N. Soliman, S. Tawab, S. Eissa, and A. Eissa, "Sero-prevalence and risk factors associated with Toxoplasma gondii infection among pregnant women in Alexandria, Egypt," International Journal of Reproduction, Contraception, Obstetrics and Gynecology, vol. 5, pp. 4220-4227, 2016.

[27] X. Huang, X. Xuan, H. Hirata et al., "Rapid immunochromatographic test using recombinant SAG2 for detection of antibodies against Toxoplasma gondii in cats," Journal of Clinical Microbiology, vol. 42, no. 1, pp. 351-353, 2004.

[28] A. J. A. C. El-Moamly, "Immunochromatographic techniques: benefits for the diagnosis of parasitic infections," Ghana Medical Journal, vol. 1, no. 4, pp. 1-8, 2014.

[29] R. Anteson, M. Techiwara, and N. MJGMJ, “Toxoplasmosis in Ghana IV," Further evidence of congenital disease caused by Toxoplasma gondii infections, vol. 19, no. 3, pp. 146-148, 1980. 